\title{
Microbiology in Dairy Processing: Challenges and Opportunities. By Palmiro Poltronieri. IFT Press Series, Wiley-Blackwell, UK, 2017; 321 pp.; ISBN: 978-1-119-11480-2
}

\author{
Franca Rossi \\ Istituto Zooprofilattico Sperimentale dell'Abruzzo e del Molise (IZSAM) "G. Caporale". \\ Specialized Diagnostic Department, 86170 Isernia, Italy; f.rossi@izs.it; Tel.: +39-0865-414619
}

Received: 13 February 2018; Accepted: 26 February 2018; Published: 28 February 2018

In this context, an overview is presented of the book "Microbiology in dairy processing; challenges and opportunities", edited by Palmiro Poltronieri, researcher at the Institute of Sciences of Food Productions of the Italian National Research Council and published by Wiley-Blackwell, for the Institute of Food Technologist (IFT) press series (doi: 10.1002/9781119115007).

This publication resulted from the collaboration of eminent academics from the Departments of Food Microbiology at several universities_from Athens, Catania, Ghent, Milan, Naples, Piacenza, Pisa, Sassari, Turin and Viçosa, scientists of the Italian National Research Council, of the Italian and Spanish institutes for Agricultural Technology and Economy, of the CREA/Zootecnia and Agricultura Institute located in Lodi, of the national veterinary control and research institute Instituto Zooprofilattico Sperimentale "G. Caporale" and the scientific director of Sacco Srl., a company in the dairy field.

Deriving from the competences of these different professionals, the book combines basic scientific concepts with applied aspects in dairy technology.

The book is a synthesis of the up to date knowledge on safety, quality, technological, nutrition and health promoting properties of dairy products and of how these are influenced by microorganisms, both harmful or beneficial, that normally constitute the microbiota of raw milk. In particular, the text outlines the new solutions to old problems affecting safety and quality of dairy products that were made possible by the advancements in the microbiological field allowed by the application of novel high throughput investigation tools such as the next generation sequencing (NGS) techniques.

The different topics are treated in a comprehensive manner providing plenty of starting points for dairy operators and researchers in this sector.

Operators of the dairy sector can find indications on how to preserve as well as possible the quality of raw milk and derived products and how to increase the health promoting properties of dairy productions with a consequent increase in product added value.

Researchers can find a wide overview of the most relevant investigation lines in the dairy field, with indications on how to advance from the state-of-the-art and offer qualified assistance to the dairy industries in the development of safe, high quality and innovative products able to convey new benefits to the consumer.

Two chapters are dedicated to the hardest to fight harmful microorganisms in dairy productions, i.e., the aerobic and anaerobic bacterial spore formers and psychotropic milk spoiling pseudomonads. Basic knowledge on their physiology, that makes them able to survive in milk and dairy products, to produce toxic compounds or enzymes that degrade milk constituents and deteriorate milk quality reducing technological exploitation, is integrated with a description of the hazards and quality defects that these bacteria can cause and with indications on how to minimize their presence and development in milk and cheese to prevent their deleterious effects. 
In the perspective of product quality safeguard is also the chapter regarding bacteriophages that infect lactic acid bacteria (LAB) causing the delay or failure of the fermentation processes. Methods to counteract this problem are presented, among which is the possibility to select phage-insensitive cultures and bacterial strains with phage adaptive immunity traits such as the Clustered Regularly Interspaced Short Palindromic Repeats (CRISPR)-cas systems.

One chapter, regarding bacteriocins from the LAB species of highest relevance for the dairy sector, indicates that these antimicrobial peptides, when produced in situ in dairy products, can inhibit pathogenic or spoiling bacteria and that better results in the inhibition of undesired bacteria can be accomplished by selecting and combining bacteriocin producing cultures. In addition, the most novel methods to identify bacteriocin producers are described.

Some chapters are dedicated to the description of the structure, activities and health implications of the chemical constituents of milk-i.e., milk fats, proteins and minerals and how microorganisms - mainly LAB - interact with those constituents to form products that can increase digestibility, nutritional value, absorption and bioavailability of vitamins and microelements and the formation of bioactive substances.

Other chapters are dedicated to LAB genetics, metabolism and activities in dairy products. These review updated knowledge on the genetic endowment and diversity of LAB that became much clearer after huge genome sequence data acquisition. Indeed, sequencing of entire genomes has now become the most straightforward approach to define aspects like the capacity to utilize substrates, have a good technological performance, tolerate environmental stresses, resist to bacteriophages, exert probiotic functions and synthesize beneficial compounds.

As a practical contribution to $L A B$ research, one chapter reports culture media and growth conditions for enumerating and isolating different LAB groups, i.e., hetero- and homo-fermenters, thermophilic and mesophilic species. Selective supplements for media and substrates that allow the growth of only some species are detailed.

The molecular methods commonly adopted for LAB typing and identification are also illustrated in a dedicated chapter.

Chapters that describe the technological processes applied to milk, the thermal treatments, fat separation and transformation and yogurt production, are included and complement those describing the microbiological aspects of dairy processing.

For the variety of themes addressed and reviewing of the most recent research and technical achievements, this recently published book can be a very useful guide to all those interested in process innovation and quality enhancement in the dairy sector. 